\title{
Modeling of ultrasound tomographic imaging for non-destructive inspection of underwater structures
}

\author{
Igor Belykh \\ Peter the Great St. Petersburg \\ Polytechnic University \\ Polytechnicheskaya, 29 \\ Russia, 195251, St. Petersburg \\ Igor.Belyh@cit.icc.spbstu.ru
}

\author{
Ivan Zakharov \\ Peter the Great St. Petersburg \\ Polytechnic University \\ Polytechnicheskaya, 29 \\ Russia, 195251, St. Petersburg \\ zakharoiv@gmail.com
}

\author{
Egor Lebedev \\ Peter the Great St. Petersburg \\ Polytechnic University \\ Polytechnicheskaya, 29 \\ Russia, 195251, St. Petersburg \\ mattspring0@gmail.com
}

\begin{abstract}
In non-destructive inspection regular ultrasound techniques provide detection and estimate size of specific defects or undesired objects in different structures. The method of tomographic imaging modeled in this paper can provide the correct position and shape of the object inside underwater structures to be inspected. The proposed method is based on 2-D full waveform inversion of reflected ultrasound field registered by the array of sensors at a short distance from the target object. The computationally intensive algorithm for tomographic image reconstruction is described and tested with focus on underwater pipeline ultrasound inspection. The results of numerical modeling using parallel calculations and physical modeling are presented and discussed.
\end{abstract}

\section{Keywords}

Ultrasound tomography, waveform inversion, physical modeling, non-destructive inspection.

\section{INTRODUCTION}

The aim of ultrasonic (US) non-destructive testing and inspection (NDI) methods is to detect the presence or absence of flaws in the form of internal defects or objects in materials, infrastructure and equipment in various industries [Bli96]. Among the NDI methods aimed at studying defects are echopulse (reflected waves), shadow (transmitted waves) and diffraction (diffracted waves). The most reliable method of ultrasound phased arrays uses lines or matrices of piezoelectric transducers to produce focused US beams for flaw detection in NDI and medicine. These methods can determine the position and estimate size of the target object, but not a detailed information about its shape, orientation and configuration. In essence, US NDI methods are aimed at revealing the structure of inhomogeneous medium from the registered wave field. However, this inverse problem solution often come down to comparing the recorded wave patterns with the referenced ones for the most frequently encountered defects, which does not always lead to satisfactory results [Zie18].

The use of ultrasound tomography (UST) has good potential for the NDI in terms of determining the detailed configuration and physical properties of the specific defects without a priori information about

Permission to make digital or hard copies of all or part of this work for personal or classroom use is granted without fee provided that copies are not made or distributed for profit or commercial advantage and that copies bear this notice and the full citation on the first page. To copy otherwise, or republish, to post on servers or to redistribute to lists, requires prior specific permission and/or a fee. them.

When elastic waves propagate in heterogeneous media, various effects distort the wave field - these are multiple reflections of waves from internal boundaries, wave front migration due to refraction, energy scattering and absorption in various materials, and also the effect of wave diffraction on inhomogeneities. Elimination of diffraction artifacts allows to more accurately determine the shape and position of objects and identify small objects in ultrasound images [Bel17]. In US phased arrays diffraction effects are minimized at physical level. Implementations of theoretical approaches to suppressing reverberations and diffraction distortions were developed for seismic data processing problems solution. These methods were popular when the computing power was not enough for a full numerical solution of the wave equation [Vir09].

\section{RECENT SOLUTIONS}

Despite current approaches to solving inverse problems by the waveform inversion method in medical applications [Li08] and NDI [Sei17] being computationally complex, they are very promising because they minimize all distorting effects, allowing to obtain correct spatial image of the internal structure of the object under study, including mapping of absorption coefficient.

Over the past decade a significant theoretical and practical progress has been achieved in solving the problems of UST for medical applications. Most 
solutions employ a toroidal transducer system to register total wave field of both refracted and reflected waves. The inspected medium has to be inside the tor, but this imposes restrictions on its size. The approaches to UST differ not only in types of the waves used, but also in methods of reconstructing spatial images from a recorded wave field. There were efforts to solve the wave equation by difference schemes in time domain [Gon17] or wave inversion in frequency domain [San15]. The above approaches of obtaining tomographic images are very computationally intensive, but are close to practical implementation [Hua16] because in recent years, massive parallel computing systems such as GPU clusters and supercomputers have been rapidly developing.

In this paper a method for obtaining an image of 2-D inhomogeneous medium is modeled by registering only reflected wave field at a certain distance from the object of interest with an array of transducers and its subsequent inversion. This acquisition scheme does not impose restrictions on the size of the object and allows to build a high-resolution volumetric ultrasound image with a set of two-dimensional tomographic slices. The paper presents the results of solving forward and inverse problems of ultrasound image reconstruction based on 2-D inversion of reflected wave field in time domain and explores the applicability of this method in non-destructive inspection technology. It is known that time-reversal reconstruction has a slightly improved signal to noise ratio [Tre14] compare to frequency-reversal one. Numerical modeling is supported by physical laboratory experiments.

\section{PROPOSED SOLUTION Forward Problem}

The forward problem is formulated for the heterogeneous elastic half-space with an array of $N$ transducers on its surface and a given shape of the pulse emitted by the source. Each transducer $s$ alternately emits a given pulse, and all $N$ transducers, including $s$, receive reflected waves from the medium during the recording time $T$.

The forward problem consists in finding a numerical solution of the wave equation in the acoustic approximation, having the form:

$\frac{1}{c^{2}(x)} \frac{\partial^{2} p\left(x, t ; x_{s}\right)}{\partial t^{2}}-\nabla^{2} p\left(x, t ; x_{s}\right)=s\left(x, t ; x_{s}\right)$,

where $x_{s}$ is the position of the pulse source, $p\left(x, t ; x_{s}\right)$ is the pressure wave amplitude at point $x$ at time $t ; c(x)$ is the speed of sound at the point $x$, $s\left(x, t ; x_{s}\right)$ is the initial signal.

There are various approaches to the numerical solution of such an equation, for example, the classical finite difference method mentioned above. Although, pseudo-spectral method [Ver18], in which spatial derivatives are approximated by the Fourier transform, is more stable and less resource-intensive. The solution of forward problem with $N$ positions of the source is a wave field in the form of a set of $N$ frames, i.e. ensembles of data received from one source, and each frame consists of $N$ records, calculated for every receiver.

\section{Inverse Problem}

The input data for the inverse problem are wave field records obtained as a result of forward problem solution or physical experiment. They are stored in the form of $N$ multichannel frames of finite time $T$. The solution of the inverse problem is spatial distribution of the acoustic parameter of the inhomogeneous medium restored from the original reflection wave field. In this case, the acoustic parameter is proportional to reflection coefficient at each point of two-dimensional space.

The main idea of the waveform inversion method is to use knowledge of the mathematical model of elastic wave propagation for numerical modeling of the physical process and finding those parameters of the equation that allow the most accurate simulation of data obtained as a result of a numerical or physical experiment.

The wave inversion problem is formulated as the problem of minimizing some objective function characterizing the deviation $\delta p$ of the simulated data from the experimental data. The objective function was taken as [Liu17]:

$$
\begin{gathered}
E(c)=\frac{1}{2} \sum_{s} \sum_{r} \int_{0}^{T}\left[\delta p\left(x_{r}, t ; x_{s}\right)\right]^{2} d t, \\
\delta p\left(x_{r}, t ; x_{s}\right)=p\left(x_{r}, t ; x_{s}\right)-p_{o b s}\left(x_{r}, t ; x_{s}\right),
\end{gathered}
$$

where $s$ is the number of the signal source; $r$ - signal receiver number; $c$ is the current velocity model; $p\left(x_{r}, t ; x_{s}\right)$ and $p_{o b s}\left(x_{r}, t ; x_{s}\right)$ are modeled and observed data received by receiver $r$ at time $t$ when emitted by source $s$.

To determine the direction of minimization at each iteration, the gradient of the function $E$ is calculated in the approximation $g^{(k)} \approx \nabla E\left(c^{(k)}\right)$, after which the current velocity model is shifted by the following formula:

$$
c^{(k+1)}=c^{(k)}+\alpha^{(k)} d^{(k)},
$$


where $c^{(k)}$ is the velocity model built at the $k$-th iteration; $\alpha^{(k)}$ is the minimization step; $d^{(k)}$ is the direction of minimization, which depends on the optimization strategy, and in the used conjugate gradients $(\mathrm{CG})$ method is defined as:

$$
d^{(k)}=-g^{(k)}+\beta^{(k)} d^{(k-1)},
$$

where $\beta^{(k)}$ is a parameter that is selected depending on the implementation. The $\mathrm{CG}$ method refers to locally optimizing, because depending on the choice of the initial approximation of the velocity model $c^{(0)}$, the algorithm can converge to an extremum that is not a global minimum of the objective function. This is especially evident when solving nonlinear problems, which include the problem of data inversion using the wave equation. The execution of each iteration of the algorithm involves the calculation of two main parameters: the gradient vector and the scalar value of the optimization step. CG method is efficient but its disadvantage is that it needs to store not only sensor data, but also the total wave field of the solutions of the main and conjugate equations that significantly increases the amount of data stored in memory.

One of the ways to overcome the instability of optimization problems is regularization, i.e. adding some additional term to the error function condition. Most often, regularization is a function that depends on some a priori data about the model, in this case, the initial distribution of velocities. For waveform data inversion, it is important that the regularizing function preserves the edges of objects in the image for greater resolution. Therefore, the total variation (TV) regularization was used. The regularized error function has the following general form:

$E_{R}(c)=E(c)+\lambda R(c)$,

where $\lambda$ is the regularization coefficient, which determines the degree of influence of the regularizing function on the result. The coefficient value is determined empirically for a specific task. TVregularization is often used for tomographic images reconstruction and its function has the following form [Lin12]:

$R_{T V}(c(x))=\sum_{x} \sqrt{\varepsilon^{2}+\|\nabla c(x)\|^{2}}$,

where $x$ is the spatial coordinate, $c(x)$ is the velocity distribution, $\varepsilon$ is small a term providing differentiability with the norm equal to zero in the entire region. In our case it was chosen as 0.01, lesser values showed worse results.

The expression for calculating the gradient of this function is as follows:

$\nabla R_{T V}(c(x))=\nabla \cdot \frac{\nabla c}{\sqrt{\varepsilon^{2}+|| \nabla c(x) \|^{2}}}$
As mentioned earlier, the calculation of the gradient by the conjugate state method is complicated by the need to read and process large amounts of data at each iteration, which with restrictions on the inputoutput system significantly increases the time for the so-called "overhead". To accelerate data inversion process, the method of ordered subsets was used [Hud94]. This method consists in dividing the whole sensor data into several sets, each of which contains a certain number of frames. For each such set, an iteration step is performed that minimizes some local error function defined separately for each set. In order for the minimizing algorithm to work correctly for small subsets, the error function was normalized with respect to the real data of the frames of each subset. As a result, much smaller amount of data is required for each iteration, which significantly speeds up the process without noticable quality reduction of the final image.

\section{EXPERIMENTAL RESULTS}

\section{Numerical modeling}

The software application for the proposed method was implemented in MATLAB medium with the kWave Toolbox [Tre14], which allows to model a forward problem solution, including ultrasound frequency range.

Sequential algorithms for solving the forward and inverse problems are computationally expensive: the steps of finding the direction and optimization step requires an additional solution of 4-5 forward problems. At the stage of calculating the gradient by the conjugate state method, it is required to preserve the amplitudes not only at the locations of the transducers, but also in the rest of the region, at every moment in time. The volume of data to be processed at each iteration for the mentioned model is more than $100 \mathrm{~GB}$. Data of such a size cannot be stored in RAM, therefore, when performing the necessary calculations, an intensive disk exchange operations occur.

The resource-intensive process of solving the forward problem was optimized by parallel computations on the GPU using CUDA technology, as well as the MATLAB Parallel Computing Toolbox. As a result of such optimization the computation speed of the program increased by approximately one order.

Due to the loaded computer resources, the program was tested on a small but acoustically representative model as a vertical cross-section of underwater pipeline filled with motor oil.

On Fig. 1 the modeled pipeline is presented in the form of sound speed distribution in $[\mathrm{m} / \mathrm{s}]$ and contains a foreign object of dense material. The size 
of the model in $\mathrm{X}$ and $\mathrm{Y}$ dimensions are $138 \times 128$ respectively in steps of computational grid for numerical solution of the equation (1). Linear size of each step is $d x=d y=0.132 \mathrm{~mm}$. The tube diameter is equal to 40 steps.

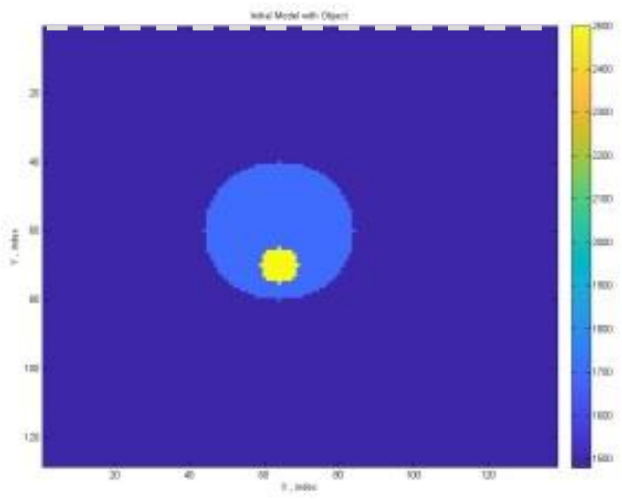

Fig.1. Initial model of underwater pipe cross-section, containing undesired object inside.

The model was probed with a single cycle pulse at a central frequency of $2 \mathrm{MHz}$. The array of 47 transducers is positioned along the $\mathrm{X}$ axis above the pipe at the upper margin of the model and is presented with a dash line. The distance between sensors is equal to 3 steps. The thickness of tube wall was considered to be much less than the wavelength and was not taken into account.

The decreasing dependency of the error function on the number of iterations in solving the inverse problem was experimentally established, which confirms the correctness of the optimizing algorithm. At 45 iterations the error function dropped down to the value of $2.3 \times 10-7$ (Fig.2).

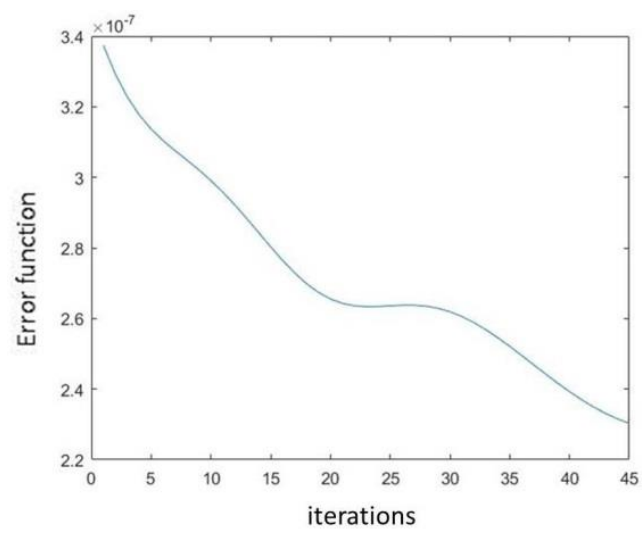

Fig.2. Error function dependency on the number of iterations.

During the inversion process the main object appears after the first iteration (Fig.3a), and the shape of small internal foreign object appears with an increase in the number of iterations (Fig.3b). Average number of iterations needed to achieve good image quality is equal to 4 . Internal object round shape is easily identifiable, although sub-vertical boundaries of the pipe are weaker than sub-horizontal ones on both restored models. This is due to sub-vertical angles of incident waves that do not produce reflections.
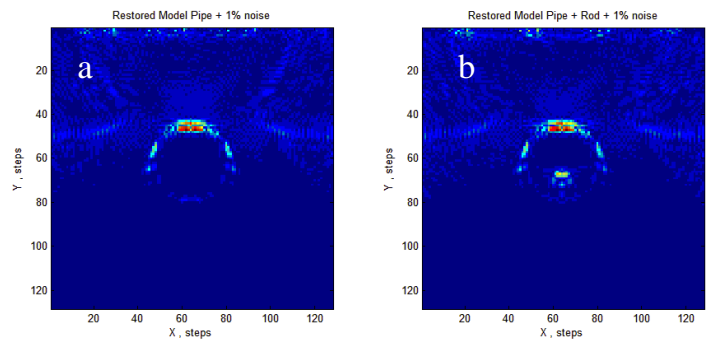

Fig.3. Reconstructed initial model without (a) and with internal object (b) with $1 \%$ of added noise.

The proposed method is quite resistant to the level of noise in the modeled wave field. With an increase in the level of additive noise from $1 \%$ (Fig. 3) to $10 \%$ (Fig. 4) the configuration of the pipe as well as the shape of internal object can be traced, although the lower wall of the tube is much less visible. With a further increase in the noise level, the quality of the reconstructed images degrades to unsatisfactory level, which imposes certain requirements to the development of acquisition equipment for nondestructive inspection based on tomographic images.
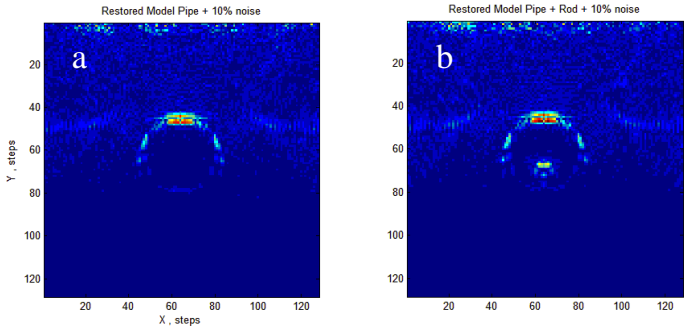

Fig.4. Reconstructed models with level of added random noise increased to $10 \%$.

The shape of initial pulse complicates the reconstruction result and requires sophisticated deconvolution methods, which are not a subject of this paper. Fig.5 shows the result of model reconstruction by the proposed method at source frequency $2 \mathrm{MHz}$ with sounding pulse having three and five cycles.
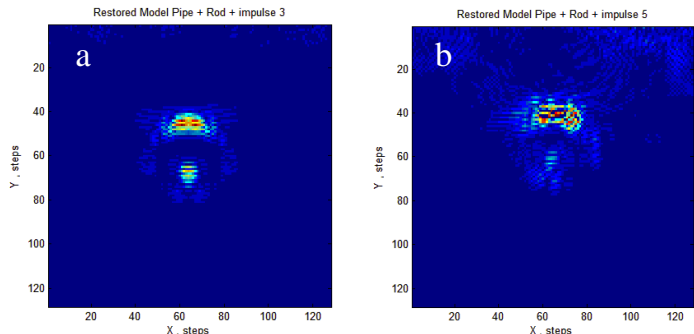

Fig.5. Restored model containing pipe and internal object with different shape of initial pulse: (a) three cycles, (b) five cycles. 
It is noticeable that more complex shape of the pulse leads to defocusing of the image and its content could be misinterpreted. Overall resolution of the implemented waveform inversion is about few grid steps, which is about half of wavelength benchmark. It is a little better in the tomographic approach compare to regular US techniques due to insonification of the medium by a vast number of waves at different angles.

For the selected model and acquisition geometry the solution of one forward problem on personal computer with 4 cores, $2.6 \mathrm{GHz}, 8 \mathrm{~GB}$ RAM, 500 GB HDD and with graphics card NVIDIA GeForceRTXsuper2060 takes less than one minute. The inverse problem is solved in about 6 minutes.

\section{Physical modeling}

Physical model was designed based on numerical model magnified by scale 10:1 since the sounding pulse of real transducers has center frequency 10 times lower, which is about $200 \mathrm{KHz}$ (Fig.6). The sizes of water tank were chosen equal to $L \times W \times D=70 \times 40 \times 30 \mathrm{~cm}$. In the first experiment, the thin plastic tube $40 \mathrm{~cm}$ long and $40 \mathrm{~mm}$ in diameter was filled with oil and was placed horizontally under the water at position $0.55 \mathrm{~L}$ in $W$ direction and at depth $D$ equal to $40 \mathrm{~mm}$. In the second experiment, a round ceramic rod $10 \mathrm{~mm}$ in diameter and $40 \mathrm{~cm}$ long was placed inside the tube at position $3 / 4$ of its diameter below the center, as shown in Fig.1.
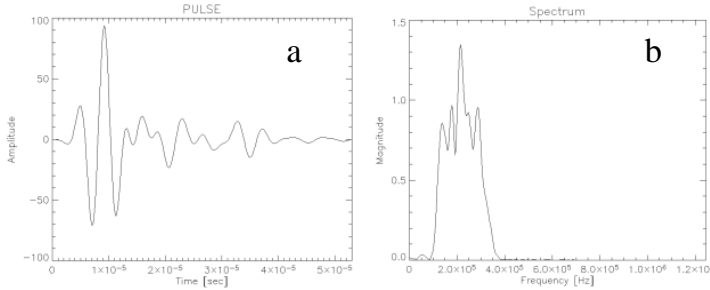

Fig.6. Source pulse (a) and its spectrum (b)

The $23 \mathrm{~cm}$ long array of 47 transducers was placed in the middle of water tank along the $L$ direction with its center position $[0.5 L, 0.5 \mathrm{~W}]$. This acquisition geometry was implemented by means of precise automized positioning equipment. Due to very stable source pulse, only two transducers were used as a source-receiver pair and the tomographic sensor array was emulated the following way. One transducer served as emitter-receiver and moved consequently through all 47 positions with $5 \mathrm{~mm}$ distance between them. Second transducer served as receiver and for each position of the source it moved through the rest 46 positions. The used transducers have very broad directionality chart, which allowed to consider the registered reflection field to be generated by quasi-point source. The spatial sizes of tank provided enough space to avoid any side or bottom reflections within the time of recoding the primary wave signals, which was equal to $T=270 \mu \mathrm{s}$.

Finally, a set of 47 frames with 47 records each was received. The set was preprocessed and used as an input for inverse problem solution. The results of experimental data inversion is presented at Fig.7.
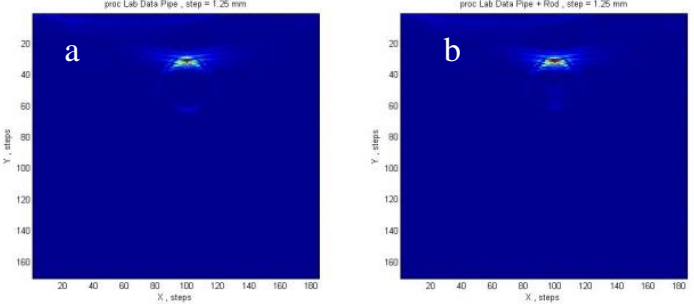

Fig.7. Reconstructed images of physical models of the underwater pipe (a), containing internal foreign object (b).

The quality of the restored images is not as good as on the numerical models described above due to the complexity of the sounding pulse, possible spatial inaccuracy in transducer positioning and level of real noise during the acquisition process. Also not all nonlinear effects are taking in account in the implemented software package. A simple thresholding procedures were applied to balance the contrast of the restored images. Some post processing efforts need be applied to suppress the noise in the final image and to make it more suitable for interpretation and decision making, but this is beyond the content of this paper. Nevertheless the shapes of the pipe and the inside object can be recognized and correct position and size of both physical objects can be retrieved.

\section{CONCLUSION}

The original algorithm was proposed for 2-D tomographic ultrasound images reconstruction based on waveform inversion of the reflected field in time domain. The proposed algorithm featured accuracy and computational efficiency and is more resistant to noise compare to finite-difference methods, however, it must be improved to meet the class of real noise in physical models and real structures. The advantage of the method is that there are no restrictions on the size of the inspected media because reflected waves can be recorded on its surface or at a distance without physical contact between sensor array and the targeted object. The resolution of tomographic images is higher than in traditional NDI approaches due to the multiple number of wave propagated through the medium at various angles. In order to obtain good image quality of the studied objects, it is necessary to select the optimal sounding frequency and shape of the emitted pulse. A volumetric image 
can be represented by a set of two-dimensional slices. These advantages will allow the proposed approach to be applied to a wider range of non-destructive testing and inspection tasks. The disadvantage of the approach on reflected waves may be the absence of reflections from the vertical boundaries of the studied objects, which may impose certain requirements on the size and geometry of the acquisition system with appropriate number of channels. The developed algorithm was implemented using GPU parallel computations, which significantly reduced the time of image reconstruction. The simplification of the spatial model of the acoustic medium consisting of objects of a relatively simple shape with nominal parameters, does not fundamentally affect the solution of the problem and is due only to computing power.

The results of numerical and physical modeling can be scaled by wavelength to achieve the required resolution in the infrastructural objects of the real size. For example, underwater oil pipeline of $1 \mathrm{~m}$ in diameter and with a wall thickness of $10 \mathrm{~mm}$ or less can be inspected without physical contact with transducers functioning at ultrasound frequency about $50 \mathrm{KHz}$ with resolution inside the pipe about $13 \mathrm{~mm}$.

The solution of the problem in 3-D is of further interest for obtaining volumetric high-resolution tomographic ultrasound images for non-destructive inspection of complex structures and equipment.

\section{REFERENCES}

[Bel17] Belykh I., Markov D. Volumetric ultrasound imaging: modeling of waveform inversion // Computer Science Research Notes 2703, 2017, P. 77-82.

[Bli96] Blitz, J. and Simpson, G. Ultrasonic Methods of Non-Destructive Testing, 1996.

[Gon17] Goncharsky A.V., Romanov S.Y., Seryozhnikov S.Y. Inverse Problems of Layerby-Layer Ultrasonic Tomography with the Data Measured on a Cylindrical Surface. // Vychislitelnyye metody i programmirovaniye. MGU, 2017, T. 18, P. 267-276, (rus).

[Hua16] Huang L., Shin J., Chen T., Lin Y., Gao K., Intrator M., Hanson K. Breast ultrasound tomography with two parallel transducer arrays // Proc. SPIE 9783, Medical Imaging 2016: Physics of Medical Imaging, 97830C-1, 2016, 12 p.
[Hud94] Hudson H. M., Larkin R. S. Accelerated image reconstruction using ordered subsets of projection data // IEEE Trans. Med. Imaging, 1994, P.100-108.

[Li08] Li C., Duric N., Huang L. Clinical breast imaging using soundspeed reconstructions of ultrasound tomography data // Medical Imaging Int. Society for Optics and Photonics, 2008, P. 692009-1-692009-9.

[Lin12] Lin Y., Huang L., Zhang Z. Ultrasound waveform tomography with the total-variation regularization for detection of small breast tumors // SPIE Medical Imaging, 2012, P. 832002-1832002-9.

[Liu17] Liu Y., Teng J., Xu T., Badal J., Liu Q., Zhou B. Effects of conjugate gradient methods and step-length formulas on the multiscale full waveform inversion in time domain: numerical experiments // Pure and Applied Geophysics, 2017, P. 1983-2006.

[San15] Sandhu G.Y., Li C., Roy O., Schmidt S., Duric N. Frequency domain ultrasound waveform tomography: breast imaging using a ring transducer // Physics in Medicine and Biology, 2015, Vol. 60, no. 14, P. 5381-5398.

[Sei17] Seidl R. Full Waveform Inversion for Ultrasonic Nondestructive Testing. PhD thesis, TECHNISCHE UNIVERSITAET MUNCHEN, 2017.

[Tre14] Treeby B. E., Jaros J., Rohrbach D., Cox B. T. Modelling Elastic Wave Propagation Using the $\mathrm{k}$-Wave MATLAB Toolbox, in IEEE Int. Ultrasonics Symposium, pp. 146-149, 2014.

[Ver18] Vereshchagin K.A., Belykh I.N. Ultrasound image reconstruction based on waveform inversion method using parallel calculation algorithms. // St. Petersburg State Polytechnic University Journal: Computer Science. Telecommunications and Control Systems. Vol. 11, No. 4, 2018, P. 49-62, (rus).

[Vir09] Virieux J., Operto S. An overview of full waveform inversion in exploration geophysics // Geophysics, 2009, P. WCC1-WCC26.

[Zie18] Zielińska M., Rucka M. Non-Destructive Assessment of Masonry Pillars using Ultrasonic Tomography. Materials 2018, 11, 2543; doi: 10.3390/ma11122543. 